\title{
ATIVIDADE OVICIDA DE EXTRATOS AQUOSOS DE MELIÁCEAS SOBRE A MOSCA BRANCA Bemisia tabaci (GENNADIUS) BIÓTIPO B EM TOMATEIRO
}

\author{
Antonio Pancrácio de Souza; José Djair Vendramim* \\ Depto. de Entomologia, Fitopatologia e Zoologia Agrícola - USP/ESALQ, C.P. 9 - CEP: 13418-900 - Piracicaba, SP. \\ *Autor correspondente <jdvendra@carpa.ciagri.usp.br>
}

\begin{abstract}
RESUMO: A mosca branca Bemisia tabaci (Gennadius) biótipo B atualmente é uma das principais pragas do tomateiro. $O$ uso de extratos botânicos no controle desse inseto é uma alternativa promissora, mas que ainda precisa ser melhor pesquisada. O presente trabalho teve como objetivo determinar a bioatividade de extratos aquosos de folhas de Melia azedarach L. e de ramos de Trichilia pallida Swartz, sobre a mosca branca Bemisia tabaci (Gennadius) biótipo B, criada em tomateiro. Foram realizados dois experimentos, avaliando-se a mortalidade e duração das fases de ovo e ninfa, após o tratamento da fase de ovo com os referidos extratos. No primeiro experimento, foram testadas as concentrações de 1 e $2 \%(\mathrm{p} / \mathrm{v})$, acrescentando-se a concentração de $3 \%$ no segundo experimento. Em todos os tratamentos houve efeito ovicida, sendo o maior valor obtido com T. pallida a $3 \%(52,32 \%)$. Nos demais tratamentos, a mortalidade variou entre 16 e $37 \%$. Os extratos aplicados sobre os ovos pouco afetaram a sobrevivência ninfal. Com exceção do tratamento com T. pallida a $2 \%$, no primeiro experimento, em que a mortalidade ninfal foi de $26,42 \%$, nos demais a mortalidade foi inferior a 16\%. Em nenhum dos experimentos foi verificada alteração na duração dos períodos de incubação e ninfal. Palavras-chave: Melia azedarach, Trichilia pallida, Bemisia tabaci, mosca branca
\end{abstract}

\section{OVICIDAL ACTIVITY OF AQUEOUS EXTRACTS OF MELIACEAE ON THE SILVERLEAF WHITEFLY FOR TOMATO}

\begin{abstract}
The silverleaf whitefly is today one of most important pests of tomato. The use of botanical extracts to control this insect is an interesting technique, but more research about it should be carried out. The objective of this work was to determine the bioactivity of aqueous extracts of Melia azedarach leaves and Trichilia pallida twigs on the silverleaf whitefly Bemisia tabaci (Gennadius) biotype B, reared on tomato. Extracts were applied to eggs, and mortality and duration of egg and nymphal stages were evaluated in two experiments. In the first experiment, extracts were tested at concentrations of 1 and $2 \%(\mathrm{w} / \mathrm{v})$ and in the second experiment the concentration of $3 \%$ was added. The ovicidal effect was observed in all treatments. The highest value was obtained with the extract of $T$. pallida at 3\% (52.32\%). For the other treatments the mortality ranged between 16 and $37 \%$. The extracts applied on eggs presented low effect on nymphs. With exception of $T$. pallida at $2 \%$, in the first experiment, where the nymphal mortality was $26.42 \%$, in the other treatments the values were lower than $16 \%$. The duration of egg and nymph periods were not affected by the extracts.
\end{abstract}

Key words: Melia azedarach, Trichilia pallida, Bemisia tabaci, silverleaf whitefly

\section{INTRODUÇÃO}

A mosca branca Bemisia tabaci (Gennadius) é considerada uma das principais pragas do tomateiro (Haji et al., 1998). Seus danos são causados diretamente pela sucção da seiva, injeção de toxinas e liberação de "honeydew" provocando a formação de fumagina, e indiretamente pela transmissão de doenças viróticas (Ohnesorge \& Rapp, 1986; Yokomi et al., 1990). Com o surgimento do biótipo B, considerado uma nova espécie (Bemisia argentifolii Bellows \& Perring) por alguns entomologistas (Bellows Jr. et al., 1994), os danos têm sido intensificados em razão da sua maior agressividade, que resulta da maior fecundidade, ampla gama de hospedeiros, alta resistência aos inseticidas e capacidade de causar desordens fisiológicas nas plantas (Costa \& Brown, 1990; Yokomi et al., 1990).

Devido ao rápido desenvolvimento de resistência dessa praga aos inseticidas (Prabhaker et al., 1998) e dos outros problemas causados por estes produtos no agroecossistema, alguns métodos alternativos têm sido estudados para o seu controle, incluindo-se, dentre eles, os extratos de plantas de diversas famílias botânicas com ênfase para as meliáceas (Coudriet et al., 1985; Asiatico \& Zoebisch, 1992; Cubillo et al., 1994; Gómez et al., 1997; Nardo et al., 1997).

O potencial inseticida de plantas da família Meliaceae tem sido avaliado, no Brasil, em relação a pragas como Spodoptera frugiperda (J. E. Smith) e Tuta absoluta (Meyrick), obtendo-se resultados promissores com algumas espécies, como Trichilia pallida Swartz e Melia azedarach L. (Rodríguez Hernández \& Vendramim, 1997; Torrecillas, 1997; Vendramim \& Scampini, 1997; Roel, 1998; Thomazini, 1999). Com essas plantas, foram obtidos, em relação a $S$. frugiperda, resultados similares 
aos registrados com Azadirachta indica A. Juss (Rodríguez Hernández, 1995; Torrecillas, 1997), considerada atualmente a mais eficiente planta inseticida (Mordue (Luntz) \& Blackwell, 1993; Rodríguez Hernández, 1995; Vendramim, 1997).

Assim, considerando a importância desse inseto na agricultura nacional e a potencialidade dos derivados vegetais de meliáceas, objetivou-se, no presente trabalho, determinar a atividade inseticida de $T$. pallida e de $M$. azedarach em relação a $B$. tabaci biótipo $B$.

\section{MATERIAL E MÉTODOS}

A pesquisa foi desenvolvida em casa de vegetação, avaliando-se os extratos aquosos de folhas de $M$. azedarach e de ramos de $T$. pallida, estruturas vegetais com atividade inseticida comprovada em relação a outras pragas (Rodríguez Hernández \& Vendramim, 1996, 1997; Torrecillas, 1997; Roel, 1998; Thomazini, 1999). Para preparo dos extratos, o material vegetal (coletado em área de mata) foi seco em estufa $\left(40^{\circ} \mathrm{C}\right.$, por 48 h) e moído até a obtenção do pó de cada estrutura vegetal, o qual foi misturado a água destilada na proporção de 1,2 ou $3 \mathrm{~g}$ por $100 \mathrm{~mL}$ de água. As suspensões foram mantidas em frascos por $24 \mathrm{~h}$ e, a seguir, filtradas através de um tecido fino ("voil"), obtendo-se os extratos aquosos a 1,2 e $3 \%$ (peso/volume), respectivamente.

A identificação da mosca branca $B$. tabaci, biótipo $B$, foi feita através da técnica RAPD-PCR (Caballero, 1996). Para a obtenção dos ovos da mosca branca, pequenas gaiolas confeccionadas por tecido de "voil" que podiam ser abertas e fechadas por uma tira de Velcroâ, foram dispostas de modo a envolver uma folha de tomateiro (com 30 dias de idade), onde foram mantidos cerca de 30 adultos não sexados do inseto. Após $24 \mathrm{~h}$, as gaiolas foram retiradas e as folhas examinadas, selecionando-se um folíolo com, no mínimo, 50 ovos. A seguir, os folíolos contendo os ovos foram pulverizados com os extratos, de modo a se obter a cobertura de toda a superfície vegetal. Como testemunha, foram utilizados folíolos com, no mínimo, 50 ovos pulverizados com água destilada. Próximo do final da fase de desenvolvimento dos insetos (o que foi caracterizado pelo tamanho das ninfas), os folíolos foram novamente envolvidos pelas gaiolas para evitar a fuga dos adultos. Para cada tratamento, foram utilizados quatro folíolos (um por planta), em delineamento experimental inteiramente casualizado. As variáveis avaliadas foram a mortalidade e a duração das fases de ovo e ninfa. Num primeiro experimento, foram testadas as concentrações de 1 e $2 \%$ dos extratos de $M$. azedarach e T. pallida, além da testemunha, acrescentando-se no segundo experimento, a concentração de $3 \%$.

As porcentagens de mortalidade $(x)$ foram calculadas para o total de insetos para cada tratamento $(\mathrm{N})$ e transformadas pela fórmula de Abbott de modo a desconsiderar a mortalidade natural (Abbott, 1925). Para comparálas, fez-se a transformação $\mathrm{y}=\operatorname{arcoseno} \sqrt{x / 100}$. A comparação de médias de y foi feita pelo teste de Tukey, com a variância teórica $V(y)=820,7 / N$ (Gomes, 1990) calculada para cada tratamento uma vez que nestes, o número de insetos foi variável.

Os dados de duração das fases de ovo e de ninfa foram analisados pelo teste $F$ e as comparações entre as médias feitas através do teste de Tukey $(P<0,05)$.

\section{RESULTADOS E DISCUSSÃO}

\section{Primeiro experimento}

A mortalidade na fase de ovo foi observada em todos os tratamentos com valores sem diferença estatística e variáveis entre 19,34 e 35,23\%. Com exceção ao tratamento com extrato de $M$. azedarach a $2 \%$, a mortalidade ninfal com aplicação dos extratos sobre os ovos foi constatada em todos os tratamentos, sendo o maior valor registrado com T. pallida a $2 \%(26,42 \%)$. A duração das fases de ovo e ninfa não foi afetada pelos tratamentos (TABELA 1).

\section{Segundo experimento}

Nesse experimento em que também foi incluída a concentração $3 \%$ das duas meliáceas, a mortalidade na fase de ovo no tratamento com extrato de T. pallida a $3 \%$ $(52,32 \%)$ foi superior às constatadas nos demais tratamentos. Nos tratamentos com extrato de T. pallida a $2 \%$ e $M$. azedarach a $3 \%$ os valores foram intermediários. Os valores mais baixos foram verificados com $M$. azedarach a 1 e $2 \%$ e com T. pallida a 1\%. A mortalidade ninfal foi relativamente baixa em todos os tratamentos não chegando a atingir $16 \%$. A duração da fase de ovo não foi afetada pelos extratos, contudo as ninfas provenientes de ovos tratados com $M$. azedarach a $1 \%$ apresentaram o período ninfal menor que as oriundas de ovos não tratados (TABELA 2).

Constatou-se alongamento do período de incubação e da fase ninfal no segundo experimento (TABELA 2) em relação ao primeiro (TABELA 1), o que provavelmente se deveu à redução da temperatura na casa de vegetação, que oscilou, em média, de $18,4^{\circ} \mathrm{C}$ para $16,6^{\circ} \mathrm{C}$, do primeiro para o segundo experimento, considerando-se que, a semelhança do que ocorre com os insetos de modo geral, a velocidade de desenvolvimento de $B$. tabaci biótipo $B$ aumenta com a redução de temperatura na faixa de 15 a $30^{\circ} \mathrm{C}$ (Wagner, 1995; Wang \& Tsai, 1996).

Alguns insetos, embora tenham completado o desenvolvimento embrionário, morreram sem conseguir romper completamente o córion do ovo. Liu \& Stansly (1995) e Prabhaker et al. (1999) também observaram esse efeito ao aplicarem, respectivamente, uma formulação a base de óleo da solanácea Nicotiana gossei e o inseticida amitraz sobre ovos de B. tabaci biótipo B. Os autores do segundo trabalho mencionaram que a morte possivelmente tenha ocorrido devido aos resíduos dos extratos depositados sobre o córion. Esta hipótese, entretanto, não pode ser utilizada para explicar a mortalidade constatada na presente pesquisa já que as ninfas mortas não chegaram a sair do ovo e, portanto, não tiveram contato com os possíveis resíduos dos extratos. 
TABELA 1 - Médias ( \pm EP) de mortalidade e duração das fases de ovo e ninfa de Bemisia tabaci biótipo B, em tomateiro, após aplicação de extratos aquosos de Trichilia pallida e Melia azedarach sobre os ovos. Temp.: $18,4 \pm 6,7^{\circ} \mathrm{C}$; UR: $72,4 \pm 21,9 \%$; fotoperíodo natural.

\begin{tabular}{|c|c|c|c|c|}
\hline \multirow[t]{2}{*}{ Extratos } & \multicolumn{2}{|c|}{ Mortalidade ${ }^{1}$} & \multicolumn{2}{|c|}{ Duração ${ }^{2}$} \\
\hline & Ovo & Ninfa & Ovo & Ninfa \\
\hline & 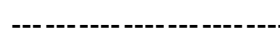 & --- & 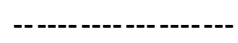 & |-- \\
\hline M. azedarach $2 \%$ & $35,23 \pm 2,65 a$ & $0,00 \pm 0,00 \mathrm{~b}$ & $7,48 \pm 0,32$ & $21,22 \pm 0,25$ \\
\hline M. azedarach $1 \%$ & $25,67 \pm 24,16 \mathrm{a}$ & $1,93 \pm 2,58 b$ & $7,48 \pm 0,35$ & $21,22 \pm 0,24$ \\
\hline T. pallida $2 \%$ & $23,19 \pm 5,02 \mathrm{a}$ & $26,42 \pm 20,25 a$ & $7,89 \pm 0,08$ & $21,24 \pm 0,23$ \\
\hline T. pallida $1 \%$ & $19,34 \pm 15,79 a$ & $8,83 \pm 11,73 a b$ & $7,94 \pm 0,03$ & $21,00 \pm 0,15$ \\
\hline Testemunha & --- & --- & $7,43 \pm 0,20$ & $20,97 \pm 0,25$ \\
\hline
\end{tabular}

Valores com letras iguais não diferem $\mathrm{P}<0,05$.

${ }^{2}$ Médias, nas colunas, não diferem pelo teste de Tukey $(P<0,05)$.

TABELA 2 - Médias ( \pm EP) de mortalidade e duração das fases de ovo e ninfa de Bemisia tabaci biótipo B, em tomateiro, após aplicação de extratos aquosos de Trichilia pallida e Melia azedarach sobre os ovos. Temp.: 16,6 $\pm 9,7^{\circ} \mathrm{C}$; UR: $80,6 \pm 22,4 \%$; fotoperíodo natural.

\begin{tabular}{|c|c|c|c|c|}
\hline \multirow[t]{2}{*}{ Extratos } & \multicolumn{2}{|c|}{ Mortalidade ${ }^{1}$} & \multicolumn{2}{|c|}{ Duração ${ }^{2}$} \\
\hline & Ovo & Ninfa & Ovo & Ninfa \\
\hline & \multicolumn{2}{|c|}{-'-} & \multicolumn{2}{|c|}{ 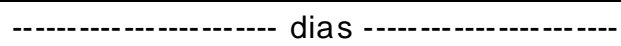 } \\
\hline M. azedarach $3 \%$ & $31,90 \pm 24,24 a b$ & $14,56 \pm 8,50 a$ & $12,56 \pm 0,56 a$ & $24,88 \pm 0,25 a b$ \\
\hline M. azedarach $2 \%$ & $17,54 \pm 14,61 \mathrm{~b}$ & $0,00 \pm 0,00 \mathrm{a}$ & $12,51 \pm 0,15 a$ & $24,88 \pm 0,36 a b$ \\
\hline M. azedarach $1 \%$ & $16,73 \pm 6,09 b$ & $6,46 \pm 5,79 a$ & $12,61 \pm 0,18 a$ & $24,69 \pm 0,35 b$ \\
\hline T. pallida $3 \%$ & $52,32 \pm 14,15 a$ & $0,00 \pm 0,00 a$ & $13,02 \pm 0,25 a$ & $25,28 \pm 0,35 a b$ \\
\hline T. pallida $2 \%$ & $36,67 \pm 8,73 a b$ & $15,41 \pm 17,70 \mathrm{a}$ & $12,74 \pm 0,28 a$ & $25,33 \pm 0,12 a b$ \\
\hline T. pallida $1 \%$ & $15,58 \pm 6,66 b$ & $7,10 \pm 9,89 a$ & $12,75 \pm 0,15 a$ & $24,87 \pm 0,40 a b$ \\
\hline Testemunha & ---- & ---- & $12,80 \pm 0,15 a$ & $25,53 \pm 0,46 a$ \\
\hline
\end{tabular}

${ }^{1}$ Valores com letras iguais não diferem $\mathrm{P}<0,05$.

${ }^{2}$ Médias seguidas de mesma letra, nas colunas, não diferem pelo teste de Tukey $(P<0,05)$.

Informações referentes ao efeito ovicida de extratos botânicos aplicados diretamente sobre ovos de Bemisia spp. são escassas na literatura, restringindo-se aos trabalhos de Coudriet et al. (1985) e Prabhaker et al. $(1989,1999)$ que registraram esse efeito sobre $B$. tabaci com o uso de nim em extrato aquoso e em formulação comercial, respectivamente.

Os extratos aplicados sobre os ovos pouco afetaram a sobrevivência ninfal. Com exceção ao tratamento com $T$. pallida a $2 \%$, no primeiro experimento em que a mortalidade ninfal foi de $26,42 \%$, nos demais a mortalidade foi inferior a 16\% (TABELAS 1 e 2). Considerando-se que a fase de ovo da mosca branca é mais difícil de ser controlada que a fase de ninfa (Coudriet et al., 1985; Prabhaker et al., 1989, 1999), os dados obtidos sugerem que, quando os insetos atingiram a fase ninfal, os compostos ativos presentes nas referidas meliáceas já se encontravam em nível residual insuficiente para afetar o inseto nessa fase.

No segundo experimento o desenvolvimento da fase ninfal foi mais rápido com aplicação do extrato de $M$. azedarach a $1 \%$ que na testemunha, o que, em princípio, foi inesperado, porque acreditava-se que, se houvesse algum efeito dos extratos, esse seria no sentido de retardar o desenvolvimento. É possível que nesse caso tenha ocorrido um efeito de hormoligose, segundo o qual agentes estressantes a um organismo numa determinada dosagem podem estimular o desenvolvimento desse organismo quando aplicado em sub-dosagem (Luckey, 1968).

Informações sobre o prolongamento da fase ninfal de $B$. tabaci por extratos de meliáceas estão restritas aos dados de Coudriet et al. (1985) que observaram esse efeito com a utilização do extrato de sementes de nim. Em relação a outras pragas, o prolongamento da fase imatura em decorrência do uso de extratos de $M$. azedarach e T. pallida têm sido referidos apenas para lepidópteros (McMillian et al., 1969; Mikolajczak et al., 1989; Chen et al., 1996; Rodríguez Hernández, 1995; Rodríguez Hernández \& Vendramim, 1997; Torrecillas, 1997; Vendramim \& Scampini, 1997; Roel, 1998; Thomazini, 1999).

\section{CONCLUSÕES}

Os extratos aquosos das meliáceas $M$. azedarach e T. pallida, em concentrações variáveis entre 1 e $3 \%$, têm efeito ovicida sobre $B$. tabaci, biótipo B. Mesmo na maior concentração, estes extratos não afetam a duração da fase imatura do inseto. 


\section{REFERÊNCIAS BIBLIOGRÁFICAS}

ABBOTT, W.S. A method of computing the effectiveness of an insecticide. Journal of Economic Entomology, v.18, p.265-267, 1925.

ASIATICO, J.M.; ZOEBISCH, T.G. Control de mosca blanca Bemisia tabaci (Gennadius) en tomate com insecticidas de origen biologico y quimico. Manejo Integrado de Plagas, v.24/25, p.1-7, 1992.

BELLOWS Jr., T.S.; PERRING, T.M.; GILL, R.J.; HEADRICK, D.H. Description of a species of Bemisia (Homoptera: Aleyrodidae). Annals of the Entomological of the Society of America, v.8, p.195-206, 1994.

CABALLERO, R. Identificación de moscas blancas. In: Hilje, L. (Ed.). Metodologías para el estudio y manejo de moscas blancas y germinivirus. Turrialba: CATIE, Unidad de Fitoprotección , 1996. cap.1, p.1-10. (Serie Materiales de Enseñanza, 37).

CHEN, C.C.; CHANG, S.J.; CHENG, L.L.; HOU, R.F. Effects of chinaberry fruit extract on feeding, growth and fecundity of the diamondback moth, Plutella xylostella L. (Lep., Yponomeutidae). Journal of Applied Entomology, v.120, p.341-345, 1996.

COSTA, H.S.; BROWN, J.K. Variability in biological characteristics, isozyme patterns and virus transmission among populations of Bemisia tabaci in Arizona. Phytopathology, v.80, p.888, 1990.

COUDRIET, D.L.; PRABHAKER, N.; MEYERDIRK, D.E. Sweetpotato whitefly (Homoptera: Aleyrodidae): Effects of neem-seed extract on oviposition and immature stages. Environmental Entomology, v.14, p.776-779, 1985.

CUBILLO, D.; QUIJIJE, R.; LARRIVA, W.; CHACÓN, A.; HILJE, $\mathrm{L}$. Evaluación de la repelencia de varias substancias sobre la mosca blanca Bemisia tabaci (Homoptera: Aleyrodidae). Manejo Integrado de Plagas, v.33, p.26-28, 1994.

GOMES, F. Curso de estatística experimental. 13.ed. São Paulo, Nobel. 430p.

GÓMEZ, P.; CUBILLO, D.; MORA, G.A.; HILJE, L. Evaluación de possibles repelentes de Bemisia tabaci: I. Productos comerciales. Manejo Integrado de Plagas, v.46, p.9-16, 1997.

HAJI, F.N.P.; ALENCAR, J.A.; PREZOTTI, L. Principais pragas do tomateiro e alternativas de controle. Petrolina: EMBRAPA, CPATSA, 1998. 50p.

LIU, T.X.; STANSLY, P.A. Toxicity of biorational insecticides to Bemisia argentifolii (Homoptera: Aleyrodidae) on tomato leaves. Journal of Economic Entomology, v.88, p.564-568, 1995.

LUCKEY, T.D. Insecticide hormoligosis. Journal of Economic Entomology, v.61, p.7-12, 1968.

MCMILLIAN, W.W.; BOWMAN, M.C.; BURTON, R.L.; STARKS, K.J.; WISEMAN, B.R. Extract of chinaberry leaf as a feeding deterrent and growth retardant for larvae of the corn earworm and fall armyworm. Journal of Economic Entomology, v.62, p.708-710, 1969.

MIKOLAJCZAK, K.L.; ZILKOWSKI, B.W.; BARTELT, R.J. Effect of meliaceous seed extracts on growth and survival of Spodoptera frugiperda (J.E. Smith). Journal of Chemical Ecology, v.15, p.121-128, 1989.

MORDUE (LUNTZ), A.J.; BLACKWELL, A. Azadirachtin: an update. Journal of Insect Physiology, v.39, p.903-924, 1993.

NARDO, E.A.B. de; COSTA. A.S.; LOURENÇÃO, A.L. Melia azedarach extract as an antifeedant to Bemisa tabaci (Homoptera: Aleyrodidae). Florida Entomologist, v.80, p.92-94, 1997.

OHNESORGE, B.; RAPP, G. Monitoring Bemisia tabaci: a review. Agriculture, Ecosystems and Environment, v.17, p.21-28, 1986.
PRABHAKER, N.; TOSCANO, N.C ;COUDRIET, D.L. Comparison of neem, ureia, and amitraz as oviposition supressants and larvicides against Bemisia argentifolii (Homoptera:Aleyrodidae). Journal of Economic Entomology, v.92, p.40-46, 1999.

PRABHAKER, N.; TOSCANO, N.C.; COUDRIET, D.L. Susceptibility of the immature and adult stages of the sweetpotato whitefly (Homoptera: Aleyrodidae) to selected inseticides. Journal of Economic Entomology, v.82, p.983-988, 1989.

PRABHAKER, N.; TOSCANO, N.C.; HENNEBERRY, T.J. Evaluation of insecticide rotations and mixtures as resistance management strategies for Bemisia argentifolii (Homoptera:Aleyrodidae). Journal of Economic Entomology, v.91, p.820-826, 1998.

RODRÍGUEZ HERNÁNDEZ, C. Efeito de extratos aquosos de Meliaceae no desenvolvimento de Spodoptera frugiperda (J.E. Smith, 1797) (Lepidoptera: Noctuidae). Piracicaba, 1995. 100p. Tese (Doutorado) - Escola Superior de Agricultura "Luiz de Queiroz", Universidade de São Paulo.

RODRÍGUEZ HERNÁNDEZ, C.; VENDRAMIM, J.D. Toxicidad de extractos acuosos de Meliaceae en Spodoptera frugiperda (Lepidoptera: Noctuidae). Manejo Integrado de Plagas, n.42, p.14-22, 1996.

RODRÍGUEZ HERNÁNDEZ, C.; VENDRAMIM, J.D. Avaliação da bioatividade de extratos aquosos de Meliaceae sobre Spodoptera frugiperda (J.E. Smith). Revista de Agricultura, v.72, p.305-318, 1997

ROEL, A.R. Efeito de extratos orgânicos de Trichilia pallida Swartz (Meliaceae) na sobrevivência e desenvolvimento de Spodoptera frugiperda (J.E. Smith, 1797) (Lepidoptera: Noctuidae). Piracicaba, 1998. 115p. Tese (Doutorado) Escola Superior de Agricultura "Luiz de Queiroz", Universidade de São Paulo.

THOMAZINI, A.P.B.W. Efeito de genótipos de Lycopersicon spp. e de extratos aquosos de Trichilia pallida Swartz (Meliaceae) sobre Tuta absoluta (Meyrick, 1917) (Lep., Gelechiidae). Piracicaba, 1999. 95p. Tese (Doutorado) - Escola Superior de Agricultura "Luiz de Queiroz", Universidade de São Paulo.

TORRECILLAS, S.M. Efeito de extratos aquosos de Trichilia pallida Swartz (Meliaceae) no desenvolvimento de Spodoptera frugiperda (J.E. Smith, 1797) (Lepidoptera) em diferentes genótipos de milho. Piracicaba, 1997. 141p. Dissertação (Mestrado) - Escola Superior de Agricultura "Luiz de Queiroz", Universidade de São Paulo.

VENDRAMIM, J.D. Uso de plantas inseticidas no controle de pragas. In: CICLO DE PALESTRAS SOBRE AGRICULTURA ORGÂNICA, 2., Campinas, 1997. Anais. Campinas: Fundação Cargill, p.64-69.

VENDRAMIM, J.D.; SCAMPINI, P.J. Efeito do extrato aquoso de Melia azedarach sobre o desenvolvimento de Spodoptera frugiperda (J.E. Smith) em dois genótipos de milho. Revista de Agricultura, v.72, p.159-170, 1997.

WAGNER, T.L. Temperature-dependent development, mortality, and adult size of sweetpotato whitefly biotype B (Homoptera: Aleyrodidae) on cotton. Environmental Entomology, v.24, p.1179-1188, 1995.

WANG, K.; TSAI, J.H. Temperature effect on development and reproduction of silverleaf whitefly (Homoptera: Aleyrodidae). Annals of the Entomological Society of America, v.89, p.375-384, 1996.

YOKOMI, R.K.; HOELMER, K.A.; OSBORNE, L.S. Relationships between the sweetpotato whitefly and the squash silverleaf disorder. Phytopathology, v.80, p.895-900, 1990.

$\overline{R e c e b i d o ~ e m ~ 25.08 .99 ~}$ 